\title{
PROGRAMA DE TUTORIA ("MENTORING"): PERCEPÇÕES DE GRADUANDOS DO CURSO DE FONOAUDIOLOGIA
}

\author{
Matheus Francoy Alpes ${ }^{1}$ \\ Larissa Donizete Corsi ${ }^{2}$ \\ Aline Epiphanio Wolf ${ }^{3}$
}

Resumo: A Tutoria ("Mentoring") é considerada uma modalidade em que uma pessoa mais experiente, denominada tutor, acompanha de perto, orienta e estimula - a partir de sua experiência, conhecimento e comportamento - um jovem iniciante em sua jornada universitária. O objetivo foi caracterizar a percepção de estudantes do primeiro semestre do Curso de Fonoaudiologia sobre um Programa de Tutoria ("Mentoring") e descrever seu funcionamento. Foi aplicado um questionário semi-estruturado construído para esta pesquisa aos alunos participantes do Programa. Os dados foram analisados quantitativamente para as questões fechadas com respostas dicotômicas ( $\operatorname{sim} /$ não) e qualitativamente através da obtenção de categorias de ocorrência para as questões abertas. Participaram 22 estudantes no total, sendo 20 (91\%) do sexo feminino e $2(9 \%)$ do sexo masculino. O intervalo de idade foi de 18 anos a 25 anos. Para as questões fechadas, o nível de concordância foi de aproximadamente $95.5 \%$. Quanto à experiência pessoal, identificamos que o Programa auxiliou no ingresso e adaptação à Universidade (08), no processo de ensino-aprendizagem (05), no funcionamento de ferramentas e webpages da Universidade (04), na resolução de problemas ou dúvidas (03) e acolhimento aos estudantes ingressantes (02). Foi um momento de troca de experiências, ideias, discussões e orientações acerca de diversos temas relacionados ao processo de ingresso e adaptação à Universidade. Os estudantes reconheceram igualmente que o Programa trouxe benefícios em âmbito pessoal, educacional e profissional, sugerindo que este deve ser mantido e aperfeiçoado ao longo dos anos.

Palavras chave: Tutoria; Universidades; Fonoaudiologia.

Abstract: Mentoring is considered a modality in which a more experienced person, called tutor, closely follows, guides and stimulates - from his experience, knowledge and behavior - a young beginner in his university journey. The objective was to characterize the students' perception of the first semester of the Speech Therapy Course on a Mentoring Program and to describe its functioning. A semi-structured questionnaire constructed for this research was applied to the students participating in the Program. Data were analyzed quantitatively for closed questions with dichotomous responses (yes / no) and qualitatively by obtaining occurrence categories for open questions. A total of 22 students participated, of which 20 (91\%) were female and 2 (9\%) were male. The age range was 18 years to 25 years. For closed questions, the level of agreement was approximately $95.5 \%$. Regarding personal experience, we identified that the Program assisted in the admission and adaptation to the University (08), in the teaching-learning process (05), in the University's tools and webpages (04), in solving problems or doubts (03) and receiving students (02). It was a moment of exchange of experiences, ideas, discussions and orientations on diverse topics related to the process of entrance and adaptation to the University.

\footnotetext{
${ }^{1}$ Faculdade de Medicina de Ribeirão Preto - Universidade de São Paulo (FMRP/USP)

${ }^{2}$ Faculdade de Medicina de Ribeirão Preto - Universidade de São Paulo (FMRP/USP)

${ }^{3}$ Faculdade de Medicina de Ribeirão Preto - Universidade de São Paulo (FMRP/USP)
} 
The students also recognized that the Program has brought benefits in a personal, educational and professional context, suggesting that it should be maintained and improved over the years. Keywords: Mentoring; Universities; Speech, Language and Hearing Sciences

\section{INTRODUÇÃO}

O ingresso à Universidade é uma transição extremamente significativa para o indivíduo pelo fato de ser um momento frequentemente sincronizado com as mudanças e adaptações peculiares da adolescência e vida adulta (POLYDORO, 2000).

O processo de entrada ao ensino repercute no desenvolvimento psicológico dos jovens estudantes. Primeiramente, representa muitas vezes a primeira tentativa importante de implementar um senso de identidade autônomo, traduzida por meio da escolha profissional (ou tentativa de escolha), que é uma tarefa típica do desenvolvimento na passagem da adolescência para a vida adulta e as experiências vividas, durante o primeiro ano na universidade são de extrema importância para a permanência no ensino superior e para obter o sucesso acadêmico (REASON et al., 2006).

O modo de integração ao contexto do ensino superior permite que eles possam aproveitar melhor (ou não) as oportunidades ofertadas pela Universidade, tanto para sua formação profissional quanto para seu desenvolvimento psicossocial. Estudantes que se integram academicamente e socialmente desde o início de seus cursos têm possivelmente mais chances de crescerem intelectualmente e pessoalmente do que aqueles estudantes que enfrentam mais dificuldades na transição à Universidade (TEIXEIRA et al., 2008).

Desenvolvido durante os anos de 1970, nos Estados Unidos, os programas de mentoring começaram a ser inseridos na área de saúde nos anos 1990, sobretudo nas escolas de enfermagem, a fim de adaptar os alunos no ambiente acadêmico, estimular na área de atuação específica e ampliar a rede de relações acadêmicas (BELLODI, 2005). Desde então, os sistemas educativos têm buscado se adaptar às novas demandas sociais, as quais exigem novas transformações nas práticas pedagógicas adotadas pelos educadores, principalmente em âmbito universitário (FRISON, 2012).

Revista Extensão em Foco, nº19, Jul./ Dez. 2019, p. 61 - 71. 
O Programa de Tutoria ("Mentoring”) visa a melhor adaptação do aluno às questões relacionadas ao novo contexto vivido pelo estudante como a saída de casa, responsabilidades pessoais, organização e/ou manutenção de estudo, inserção profissional, entre outras (PANÚNCIO-PINTO e COLARES, 2015). Normalmente ocorre no próprio ambiente universitário e têm um professor ou profissional da área como tutor (OLIVEIRA et al., 2013).

As bases preliminares que originaram a proposta de implantação de um Programa de Tutoria na Faculdade XXXXX, surgiram através de discussões internas entre a Comissão de Graduação da XXXX e do XXXX da referida Instituição. Dessa forma, em 2003, houve a implementação de um projeto "piloto" aos alunos do Curso de Medicina, a fim de identificar suas motivações para a Universidade e à profissão e dirigi-las adequadamente em âmbito pessoal, acadêmico e profissional (MARTINS, 2016).

Atualmente, os outros cursos da Unidade (Fonoaudiologia, Fisioterapia, Nutrição e Metabolismo, Informática Biomédica, Ciências Biomédicas e Terapia Ocupacional) também fazem parte da Tutoria. No caso do curso de Fonoaudiologia, o Programa foi implantado em 2009 e desde então, vêm sido descritos benefícios aos alunos submetidos a este processo.

Sendo assim, o objetivo do estudo foi caracterizar a percepção de graduandos do Curso de Fonoaudiologia sobre a experiência em um Programa de Tutoria ("Mentoring").

\section{MATERIAIS E MÉTODOS}

Estudo descritivo-exploratório, adotando abordagem quali-quantitativa. O projeto foi submetido e aprovado pela Comissão de Graduação da XXX e pelo Comitê de Ética do XXX sob n³925/2017.

Revista Extensão em Foco, nº19, Jul./ Dez. 2019, p. 61 - 71. 
O caráter exploratório deste estudo levou à construção de um instrumento semi-' estruturado. Estudantes do primeiro semestre do curso de Fonoaudiologia da FMRP-USP no ano de 2017 foram abordados por meio de questionário, formado por questões fechadas e também por questões abertas.

Em termos quantitativos nas questões fechadas dicotômicas (sim/não) foi utilizada a frequência simples para cada uma das respostas. Em termos qualitativos, utilizamos a análise de conteúdo como estratégia, através de categorização por semelhança de resposta a cada uma das questões abertas (BARDIN, 1977,p.42).

\section{RESULTADOS}

Os encontros foram realizados no ano de 2017 e teve seu início no mês de março na primeira semana de aula dos alunos ingressantes do Curso de Fonoaudiologia (Semana de Recepção aos calouros da XXXX). Os encontros eram quinzenais, com duração de uma hora aproximadamente e com horário exclusivo, visando não atrapalhar a rotina acadêmica dos participantes e também propiciar a oportunidade de maior aderência por parte dos alunos. No total foram sete encontros realizados no período e os temas eram definidos de acordo com a demanda trazida pelos próprios participantes.

\section{Caracterização dos encontros}

\section{Primeiro encontro - 06 de março de 2017}

No primeiro encontro, houve a participação de 28 alunos. Foi realizada a apresentação do Programa de Tutoria, elencando seus pontos primordiais e sua estruturação em grupo, sendo apresentadas as datas das reuniões, bem como o horário. Essas ficaram marcadas quinzenalmente, às segundas-feiras entre meio dia e treze horas.

Cada um dos alunos realizou sua apresentação pessoal, elencando seu nome, idade, cidade de origem, razão de ter escolhido o curso de Fonoaudiologia e hobbies/atividades de lazer; foi um momento de descontração, conhecimento de particularidades de cada um dos alunos e integração entre veteranos e calouros;

Revista Extensão em Foco, nº19, Jul./ Dez. 2019, p. 61 - 71. 
Os alunos trouxeram expectativas particulares sobre a Universidade e o Curso e a docente tutora, juntamente com o pós-graduando tutor e os alunos peers, promoveram uma dinâmica com a participação de todos os envolvidos que visou a interação dos mesmos e para ajudar no processo de socialização e criação de vínculos.

\section{Segundo encontro - 20 de março de 2017}

No segundo encontro, 25 estudantes participaram. Os temas abordados foram os pontos positivos e negativos da Semana da Recepção dos Calouros, sentimentos em relação ao início da graduação e organização pessoal durante as atividades acadêmicas. Posteriormente, foi aplicado um questionário proposto pelo XXX para verificar as opiniões pessoais dos estudantes sobre a Semana de Recepção.

\section{Terceiro encontro - 03 de abril de 2017}

Participaram 27 alunos que elencaram que uma das disciplinas do Ciclo Básico ministrada no semestre estava mais didática do que a anterior, após conversa com o docente responsável. Não pautaram questões maiores referentes à inadaptações e/ou dificuldades nas semanas anteriores. Conforme demanda da semana anterior, os alunos peers trouxeram temas referentes ao cotidiano da graduação: plataformas digitais da Faculdade: site da XXX e Moodle; e bolsas do Serviço de Assistência Social.

\section{Quarto encontro - 17 de abril de 2017}

Participaram de 23 alunos. Alguns dos estudantes compareceram à Semana da Voz do HCFMRP-USP e relataram ter sido uma ótima experiência inicial. A partir de pedidos dos alunos, o Tutor fez uma apresentação sobre Iniciação Científica, elencando possibilidades e formas de ingresso, bem como o oferecimento de bolsas/auxílios.

\section{Quinto encontro - 08 de maio de 2017}

Participaram 21 alunos, que levantaram questões referentes às disciplinas do Ciclo Básico, elencando que estas são densas e que estavam apresentando dificuldades frente a forma que estavam sendo ministradas e às formas de avaliação empregadas. Os Tutores orientaram os Revista Extensão em Foco, nº19, Jul./ Dez. 2019, p. 61 - 71. 
estudantes e fizeram anotações para levar as reivindicações a serem discutidas durante a próxima reunião da Comissão Coordenadora do Curso. O Tutor apresentou aos alunos através do Sistema XXXX como funcionam as inscrições do Programa de Bolsas Unificadas da Instituição e os processos de inscrição para pleitear uma das bolsas.

\section{Sexto encontro - 15 de maio de 2017}

Participaram 20 alunos. Foi realizada uma breve apresentação pelos Tutores sobre a Plataforma Lattes e a importância de sua criação para a carreira acadêmica. Com oferecimento da psicopedagoga do XXX, foi realizada uma atividade sobre estilos de aprendizagem, a fim de auxiliar os alunos a encontrarem estratégias de estudos na Universidade.

\section{Sétimo encontro - 29 de maio de 2017}

Foi realizada a Confraternização da Tutoria, onde participaram todos os alunos ingressantes, tutores e peers dos 07 cursos da XXXX no ano de 2017. Foi realizado um levantamento frente às atividades realizadas por cada um dos grupos, bem como, pontos positivos e negativos e sugestões para o próximo ano de realização do Programa.

\section{Aplicação do questionário}

No último encontro do Programa, foi aplicado o questionário com os estudantes participantes, com o intuito de especificar e verificar a experiência pessoal de cada um quanto à Tutoria e traços positivos e negativos apontados a partir de sua participação.

Participaram 22 estudantes no total, sendo 20 (91\%) participantes do sexo feminino (91\%) e 2 (9\%) do sexo masculino, como demonstrado no Gráfico 1.

\section{$>$ INSERIR GRÁFICO $1<$}

O gráfico 2 demonstra a média de idade dos participantes do programa. O intervalo de idade foi de $<18$ anos a $>$ de 25 anos. Os resultados mostram que 05 (22,5\%) participantes tinham menos de 18 anos, $14(63,5 \%)$ tinham entre 18 e 20 anos, $1(4,5 \%)$ tinha de 20 a 22 anos e 2 $(9,5 \%)$ tinham idade superior a 25 anos.

Revista Extensão em Foco, nº19, Jul./ Dez. 2019, p. 61 - 71. 


\section{$>$ INSERIR GRÁFICO 2<}

No gráfico 3, podemos observar as respostas apresentadas pelos participantes nas questões de ordem simples. O questionário contava com 08 questões fechadas do tipo dicotômica, nas quais as possibilidades de respostas seria sim ou não. As questões realizadas foram as seguintes:

Q1. Trouxe algum tipo de benefício para você?

Q2. Ajudou no processo de ingresso e adaptação à Universidade?

Q3. Beneficiou a resolução de questões e/ou problemas relacionados à universidade?

Q4. Influenciou na manutenção de estratégias de estudos/aprendizagem das disciplinas?

Q5. Os conteúdos propostos foram adequados?

Q6. Os profissionais (professores e/ou colaboradores) eram preparados?

Q7. Após o término, evidenciou benefícios a partir da experiência?

Q8. É um dever da Universidade oferecer a Tutoria Acadêmica aos alunos?

\section{>INSERIR GRÁFICO 3<}

A questão aberta do questionário foi referente à experiência pessoal quanto à participação no Programa pelos estudantes. As respostas foram analisadas e categorizadas de acordo com a sua semelhança de resposta e frequência, sendo: ingresso e adaptação à Universidade (08), processo de ensino-aprendizagem (05), funcionamento de ferramentas e webpages da Universidade (04), resolução de problemas ou dúvidas (03) e acolhimento aos estudantes ingressantes (02), conforme o Quadro 1.

\section{$>$ INSERIR QUADRO $1<$}

\section{DISCUSSÃO}

Ao longo dos séculos, o tutor assumiu papéis e valorização diferenciados. No período que antecedeu a criação do ensino superior, a tutoria era exercida nas comunidades tribais com a Revista Extensão em Foco, nº19, Jul./ Dez. 2019, p. 61 - 71. 
finalidade de ensinar as estratégias de sobrevivência e as práticas culturais e religiosas. Nos primórdios da civilização, o tutor assumia a formação moral do aprendiz (ALVERGA, 2014).

À exemplo do que acontece no Programa Institucional de Tutoria Mentoring da XXX, a oferta de espaços para o desenvolvimento de atividades práticas reflexivas, integrando professores e estudantes, torna possível o compartilhamento, a discussão, a reflexão sobre a vida acadêmica e a construção do papel profissional. Os serviços de apoio configurados na forma de assistência, apoio ao ensino e pesquisa também podem colaborar com a IES na construção de estratégias institucionais para a conquista de avanços no ensino de graduação e consequentemente na formação profissional (PANÚNCIO-PINTO e COLARES, 2015).

O processo tutorial é presencial, consistindo de um para a formação pessoal e profissional do estudante universitário. O crescente ingresso de estudantes à Universidade, os avanços tecnológicos, as dificuldades de adaptação dos alunos aos horários, as relações interpessoais, a estrutura universitária, a distância física dos campi, entre outros, têm despertado nos meios acadêmicos a necessidade de ampliar as formas convencionais de trabalhar a educação (EMERENCIANO et al., 2004).

Durante os encontros, os participantes traziam demandas referentes às expectativas pessoais relacionadas com o curso e com o "movimento universitário", ao qual se encontravam submetidos. Muitas vezes chegaram a relatar momentos de medo e insegurança diante do novo e das inúmeras informações repassadas pela instituição e pelos próprios colegas veteranos geradoras de confusão em meio à quantidade de novidades acadêmicas e pessoais - bem como, pelas dificuldades de organização e adaptação à rotina de estudos e aos métodos de avaliação propostos. Nesse momento, os tutores e peers foram fundamentais no sentido de acalmá-los e expor a sua experiência durante a graduação, os caminhos percorridos e dicas acerca de demonstrar que o período de adaptação requer paciência e organização e que as capacidades e limitações de cada um devem ser respeitadas e colocadas como elementos importantes da "nova vida" (DINIZ e ALMEIDA, 2006).

Uma pesquisa recente teve como objetivo compreender a experiência vivida por alunos de Medicina na atividade de Tutoria de uma Universidade pública do Brasil. Os elementos experienciais revelaram três conjuntos temáticos: o contexto da tutoria, o vivido na tutoria e a avaliação da experiência. A relação de tutoria mostrou contribuir tanto no enfrentamento das Revista Extensão em Foco, nº19, Jul./ Dez. 2019, p. 61 - 71. 
vicissitudes da formação quanto no exercício de habilidades como: a escuta, a aceitação e a comunicação, fundamentais para um profissional da saúde em formação (MARTINS e BELLODI, 2016).

Um estudo realizado na mesma Unidade por tutores do Programa, evidenciou que a facilidade de comunicação e o estabelecimento de vínculos interpessoais contribuíram decisivamente para o estabelecimento de forte relação de confiança e cordialidade entre todos os envolvidos, fazendo com que a Tutoria gerasse um ambiente propício para trocas de experiências e discussões acerca de temas que envolvem o âmbito pessoal, profissional e social dentro e fora da Universidade (ALPES e WOLF, 2018). Neste estudo, foi possível observar que os atores fundamentais do processo, os próprios estudantes, demonstraram ter se beneficiado de modo significativo pela participação no programa de Tutoria, relatando, por vezes, a sensação de alívio diante das pressões características dessa fase da vida universitária.

Um possível viés do estudo seria o fato de que o instrumento utilizado foi construído para a finalidade desta pesquisa e não passou por um processo de pré-teste e/ou validação, o que pode influenciar em resultados com menor inferência estatística e confiabilidade. Este fato pode ser minimizado devido à busca na literatura acerca do tema para sua construção e também devido ao alto índice de concordância de respostas entre os participantes nas questões que compõem o questionário.

Na educação de cursos da área da saúde a tutoria é importante, sobretudo para aprimorar o suporte acadêmico, reduzir o risco de burn-out e promover o desenvolvimento de relações dentro da profissão e da satisfação com a futura carreira. $\mathrm{O}$ baixo número de publicações de relatos de grupos tutoriais das escolas médicas brasileiras provavelmente é decorrente da realização de processos informais ou mesmo pela não divulgação dos projetos formais em execução ou já finalizados. O compartilhamento das experiências pode ser importante no processo de instauração, aperfeiçoamento e superação, podendo contribuir para que um maior número de alunos tenha acesso a esta ferramenta de apoio (CHAVES, 2014).

\section{CONCLUSÃO}

O Programa de Tutoria no Curso de Fonoaudiologia da FMRP-USP no ano de 2017 contou com sete encontros e foi mediado por profissionais com expertise na área.

Revista Extensão em Foco, nº19, Jul./ Dez. 2019, p. 61 - 71. 
A partir da aplicação do questionário estruturado, pode-se concluir que o Programa trouxe benefícios aos alunos participantes em âmbito pessoal, educacional e profissional, sugerindo que este deve ser mantido e aperfeiçoado ao longo dos anos.

Destaca-se a necessidade de um maior número de publicações referentes a Programas de Tutoria desenvolvidos em todo o país para que haja melhor fundamentação, aprimoramento e aperfeiçoamento dos programas já existentes, fortalecendo a implementação de novos programas em escolas da saúde, levando esta ferramenta de apoio a maior número de universitários, contribuindo para o enriquecimento de sua jornada acadêmica.

\section{REFERÊNCIAS}

ALPES, M. F; WOLF, A. E. Tutoria Acadêmica ("Mentoring"): relato de experiência de um tutorado a tutor. Revista Extensão em Foco, nº 16, Jul./ Set. (2018), p.90 -98.

ALVERGA, M. F. R. N. A. construção da autonomia no uso das TIC: avaliar o impacto das tutorias entre pares no $10^{\circ}$ ciclo. Dissertação de Mestrado em Ciências da Educação. Escola Superior de Educação e Ciências Sociais. Instituto Politécnico de Leiria. 2014.

BELLODI, P. L. \& DE ARRUDA MARTINS, M. Tutoria: mentoring na formação médica, Casa do Psicólogo. 2005.

CHAVES, L.G. a Tutoria como Estratégia Educacional no Ensino médico. Revista Brasileira de Educação Médica. 38 (4) : 532-541; 2014.

DINIZ, A. M; ALMEIDA, L. S. Adaptação à universidade em estudantes de primeiro ano: Estudo diacrónico da interacção entre o relacionamento com pares, o bem-estar pessoal e o equilíbrio emocional. Análise Psicológica, 1(XXIV), 29-38, 2006.

EMERENCIANO, M., SOUZA, C. \& FREITAS, L. Ser presença como educador, professor e tutor.(citado em: 23 set 2004). URL: http://www. abed. org. br/publique/cgi/cgilua. exe/sys/start. htm.

FRISON, L. M. B. 2012. Tutoria entre estudantes: uma proposta de trabalho que prioriza a aprendizagem. Revista Portuguesa de Educação, 25, 217-240.

MARTINS, A. D. F. \& BELLODI, P. L. Mentoring in medical students: a humane and developmental experience. Interface-Comunicação, Saúde, Educação. 2016; 20, 715726.

OLIVEIRA, I. L. L., GUIMARÃES, S. U. \& ANDRADE, J. A. A. 2013. Programa de apoio ao calouro: um enfoque na aprendizagem de adultos em um sistema de mentoria.

PANÚNCIO-PINTO MP, COLARES MFA. O estudante universitário: os desafios de uma educação integral. Medicina (Ribeirão Preto) 2015;48(3):273-81.

Revista Extensão em Foco, nº19, Jul./ Dez. 2019, p. 61 - 71. 
POLYDORO, S. A. J. O trancamento de matrícula na trajetória acadêmica do universitário: condições de saída e de retorno à instituição. 2000.

REASON, R. D., TERENZINI, P. T. \& DOMINGO, R. J. First things first: Developing academic competence in the first year of college. Research in Higher Education. 2006; 47, 149-175.

TEIXEIRA, M. A. P., DIAS, A. C. G., WOTTRICH, S. H. \& OLIVEIRA, A. M. Adaptação à universidade em jovens calouros. Psicologia escolar e educacional, 2008; 12, 185-202.

Revista Extensão em Foco, nº19, Jul./ Dez. 2019, p. 61 - 71. 
Revista Extensão em Foco, nº19, Jul./ Dez. 2019, p. 61 - 71. 